\title{
Docentes de inglés que dejan huellas. Relatos de vida
}

\author{
English teachers who leave traces. Life stories \\ Professores de inglês que deixam rastros. Histórias da vida
}

\author{
Lenin Byron Mendieta Toledo \\ lenin.mendietat@ug.edu.ec \\ https://orcid.org/0000-0002-8385-898X
}

Universidad Guayaquil, Guayaquil-Ecuador

\section{Laura Iliana Mariscal Touzard \\ laura.mariscalt@ug.edu.ec \\ https://orcid.org/0000-0002-7418-2797}

Universidad Guayaquil, Guayaquil-Ecuador

\section{Martha Amelia Castillo Noriega \\ martha.castillon@ug.edu.ec \\ https://orcid.org/0000-0002-7867-7463}

Universidad Guayaquil, Guayaquil-Ecuador

Artículo recibido en junio 2021, revisado en julio 2021, arbitrado en agosto 2021 y publicado en septiembre 2021

\section{RESUMEN}

El estudio tuvo como objetivo interpretar el perfil del profesorado universitario de inglés de Ecuador con una mirada sociohistórica a partir de las narrativas y experiencias de los docentes en relación con su formación docente, sistemas de creencias, y valores axiológicos. Este fue un estudio de carácter exploratorio, cualitativo, interpretativo, hermenéutico y de corte transversal, la población fue escogida a través de la aplicación de encuestas a estudiantes de 6 universidades. Se realizaron relatos de vida para conocer aspectos importantes de los sujetos de estudio, los mismos que se bordaron con los relatos de sus colegas y alumnos, todos recolectados a través de entrevistas semiestructuradas $y$ en profundidad. La interpretación de estos resultados demostró que estos profesores son personas humanas con defectos y virtudes, pero, sobre todo, con un sello sublime y pasión por enseñar. Se concluyó que los docentes tienen una sólida formación profesional y su estatus como profesores es el resultado de sus continuas asistencias a seminarios, talleres, maestrías y doctorados.

Palabras clave: Docentes huella; Formación; Sistema de creencias; Valores; Relatos de vida

\section{ABSTRACT}

The article was part of the national research project "The profile of English teachers in universities in Ecuador. Interpretive study of their good practices and training, teachers who leave traces". The objective was to interpret the profile of university English teachers in this country with a socio-historical perspective based on the narratives and experiences of teachers about their teacher training, belief systems, and axiological values. This was an exploratory, qualitative, interpretative, hermeneutical, and cross-sectional study, the population was chosen by applying surveys to students from six universities in Ecuador. Life stories were essential when it came to knowing essential aspects of the study subjects, which embroidered with the stories of their colleagues and students, all collected through semi-structured and in-depth interviews. Overall results of this study showed that teacher agency has built in these English teachers this sense of acting decisively and fruitfully to direct their professional growth and share their passion for teaching.

Key words: Teachers who leave a trace; Professional development; Belief system; Values; Life stories

\section{RESUMO}

$\mathrm{O}$ artigo fazia parte do projeto nacional de pesquisa "O perfil dos professores de inglês nas universidades do Equador. Estudo interpretativo de suas boas práticas $\mathrm{e}$ formação, professores que deixam rastros". $\mathrm{O}$ objetivo foi interpretar o perfil dos professores universitários de inglês neste país com uma perspectiva sócio-histórica a partir de narrativas e experiências de professores sobre sua formação docente, sistemas de crenças e valores axiológicos. Trata-se de um estudo exploratório, qualitativo, interpretativo, hermenêutico e transversal, cuja população foi escolhida por meio da aplicação de pesquisas a alunos de seis universidades do Equador. As histórias de vida foram essenciais para o conhecimento de aspectos essenciais dos sujeitos do estudo, que se bordaram com as histórias de seus colegas e alunos, todas coletadas por meio de entrevistas semiestruturadas e em profundidade. Os resultados gerais deste estudo mostraram que a agência do professor construiu nesses professores de inglês esse senso de agir de forma decisiva e frutífera para direcionar seu crescimento profissional e compartilhar sua paixão pelo ensino.

Palavras-chave: Professores que deixam rastros; Desenvolvimento profissional; Sistema de fé; Valores; Histórias da vida 


\section{INTRODUCCIÓN}

Este artículo se inscribe en el proyecto nacional de investigación "El perfil del profesorado de inglés en universidades de Ecuador. Estudio interpretativo de sus buenas prácticas y formación, maestros que dejan huellas".

El problema de investigación radica en la no existencia de un perfil del profesorado de inglés en las universidades de Ecuador, en los perfiles de egreso de las carreras para profesores de inglés que tienen las universidades, constan perfiles de egreso de los profesores que impartirán clases en escuelas y colegios, más no en universidades (Universidad Católica de Santiago de Guayaquil, 2018; Universidad Nacional de Educación, 2021; Universidad Técnica Particular de Loja, 2020). A pesar de esto, para el estudio, buscar y entramar el perfil docente se convirtió en la más apasionante oportunidad de descubrir las dimensiones que hacen del maestro de inglés un sujeto distinto dentro del contexto educativo universitario.

La UNESCO (1995) con su Documento de política para el cambio y el desarrollo en la educación superior identifica a la nueva universidad como el lugar de formación de alta calidad donde se eduque a los alumnos para proceder de modo eficaz en diferentes funciones y prácticas cívicas y profesionales, incluyendo las más desiguales, reales y especializadas.

En este sentido, la educación superior ecuatoriana, inmersa en las nuevas evoluciones educativas argumenta la docencia como una de sus funciones sustantivas. El Consejo de Educación Superior (2017) en el Reglamento de Régimen Académico sostiene que la docencia es la cimentación de conocimientos y perfeccionamiento de capacidades yhabilidades, mediantelainteracción entre docentes y pupilos para la formación integral de ciudadanos profesionales ligados con el beneficio, contribución y mejoramiento de su entorno. Esta premisa conlleva entonces a una reinterpretación del rol del educador.

La enseñanza de inglés en educación superior tuvo en el año 2011 la visión de algunos delegados de universidades para crear los estándares de enseñanza de este idioma. En el año 2015, después de casi cuatro años de trabajo, se estableció el programa de enseñanza de inglés general para el tercer nivel de educación superior. Para el año 2019, de acuerdo con lo establecido en el artículo 80 del Reglamento de Régimen Académico del Consejo de Educación Superior (2017), demandó en las carreras de pregrado el nivel B1 de acuerdo con el Marco Común Europeo de las Lenguas como suficiencia en una lengua extranjera para requisito para término de estudios.

El Documento de Política para el Cambio y el Desarrollo en la Educación Superior, publicado por la UNESCO en 1995, y que mantiene plena vigencia, reconoce que la nueva universidad debe convertirse en una comunidad dedicada plenamente a la investigación, la creación y la difusión del conocimiento, al progreso de la ciencia, y que participe en el desarrollo de innovaciones e invenciones tecnológicas (Unesco, 1995).

La Educación Superior ecuatoriana, inmersa en las nuevas transformaciones educacionales, sustenta la necesaria integración entre lo académico, lo vinculativo y lo investigativo, como eje conductor del proceso para el tránsito hacia la excelencia universitaria en la formación profesional. Una educación basada en la necesaria formación prospectiva y problémica que conlleve a la formación, de un profesional competente, comprometido socialmente y con las capacidades necesarias, para proponer modelos sociales basados 
en la integración de conocimientos y con marcado carácter humanista y científico.

En tal sentido, se propuso como objetivo de investigación interpretar el perfil del profesorado universitario de inglés en Ecuador con una mirada sociohistórica a partir de las narrativas y experiencias de los propios docentes en relación con su formación docente, sistemas de creencias y valores axiológicos.

Se destaca del presente estudio la relevancia que se le otorga a la educación superior en Ecuador, pues se sustenta en los fundamentos socioeconómicos y culturales reconocidos por el Estado, dentro del marco del PNBV y respondiendo a un entorno y contexto determinados, para lograr altos niveles de eficiencia y pertinencia en la formación de los graduados.

Ante estas necesidades sociales la educación superior ecuatoriana deberá formar personas críticas, con conciencia de los problemas que afectan a la sociedad y sus miembros con entrenamiento en técnicas de discusión y debate, capaces de criticar los propios modelos sociales en los que se les forma $\mathrm{y}$ de proponer modelos sociales superiores.

Los profesores de inglés en las universidades de Ecuador no cuentan con un perfil que los caracterice dentro de la docencia universitaria (Universidad Católica de Santiago de Guayaquil, 2018; Universidad Nacional de Educación, 2021; Universidad Técnica Particular de Loja, 2020), aunque existe un estudio que da cuenta de los perfiles de los profesores universitarios de las carreras de educación (Alfaro Rojas y Alvarado Cordero, 2018), sin embargo, como dato alentador, se divisa la construcción de perfiles de egreso para docentes que impartirán clases en escuelas y colegios (Mensias Pérez, 2016).

La construcción del perfil del docente de inglés en el nivel superior es una tarea por demás apasionante, el mayor reto fue tejer las urdimbres y tramas que constituyen al sujeto llamado docente de forma tal que no se produzcan dislocaciones en la idea central de nuestro estudio. El perfil docente universitario de inglés es un tema no investigado en Ecuador, si bien es cierto, existen investigaciones similares en otros países. Por tal razón, es un trabajo inédito, en sí el sujeto es inédito (Carpio, 2004) aunque tenga características que lo hagan ser común a los otros (Murillo, 2020).

El docente de inglés es un transformador de la realidad social de los estudiantes, es capaz de ofrecer el aprendizaje de un idioma que se ha convertido muy necesario en el contexto global, idioma que es hablado como segunda lengua por más del $20 \%$ de la población mundial, el más hablado en el mundo en cuanto a países y el tercero en relación con la población total (BBC, 2020). El profesor de inglés realiza la enculturación de forma implícita mientras enseña el idioma, a pesar de ello, es tan poco lo que conocemos de los "Teachers" como coloquialmente se los llama en Ecuador, que interpela el visitar sus territorios y conocer a fondo el perfil de estos profesores. Esta búsqueda se realizó desde el encuentro de diversas aristas que forman su arquetipo, es decir, desde una cosmovisión multidimensional en donde entran en Inter juego su formación docente, sistema de creencias $y$ valores; así también, el entramado de sus prácticas educativas.

\section{Visiones teóricas}

Mizell (2010) estipula que la formación docente es considerada como el resultado de todas aquellas experiencias educativas que son puestas de manifiesto por el docente, lo cual permiten al estudiante aprender y adquirir habilidades, destrezas y competencias que en el futuro serán puestas en práctica en su desempeño profesional, en el campo 
de la enseñanza. Si bien es cierto que muchos de los docentes de inglés vienen de diversas profesiones ajenas al de la docencia, es propio resaltar que en muchos de los casos, estos profesionales ejercen la docencia de forma proba y excelsa, todo esto, producto de su formación continua. La calidad de la enseñanza es uno de los factores más importantes para mejorar el desempeño y elevar el nivel de suficiencia de sus alumnos en esta lengua extranjera. Hervis (2018) argumenta que la calidad está asociada al desempeño docente, sin embargo, en América Latina, se presenta como una situación amorfa en cuanto a esas relaciones, el autor argumenta que, en nuestra región existen abismos entre equidad y acceso a la educación, como consecuencia de esto la calidad se ve menguada y por tanto el desempeño se hace presente como un fantasma que subyace enterrado en factores de orden político y por supuesto económico y culturales, en sí son relaciones de Biopoder (Flores, 2014).

La formación profesional y continua del docente de inglés es clave en el éxito educativo. Los requerimientos de las sociedades van cambiando constantemente, se produce una dialéctica interminable, en cuanto al aprendizaje del idioma inglés, en Ecuador de acuerdo con el Art. 80 del Reglamento de Régimen Académico del Consejo de Educación Superior "En las carreras de tercer nivel, de grado, se entenderá por suficiencia en el manejo de una lengua extranjera al menos el nivel correspondiente a B1 del Marco Común Europeo de referencia para las Lenguas" (Consejo Educación Superior, 2019). Por tanto, se genera la exigencia de que el docente de inglés posea al menos un nivel B2, además, como parte de su perfil profesional, debe poseer una maestría a fin a la enseñanza de inglés, por último, es muy importante que posea años de experiencia en docencia universitaria.
El artículo 60 de la Ley Orgánica de Educación Superior, la cual fue publicada por Secretaría de Educación Superior, Ciencia, Tecnología e Innovación. (2010), señala que uno de los dines de la universidad consiste en la formación profesional, técnica y científica de sus estudiantes, docentes e investigadores, todo bajo el enfoque humanista.

El perfil del docente debe tener un enfoque en las competencias del docente en general (Tobón, Sánchez, Carretero, y García, 2006; Tobón, 2008) y específicas del área de enseñanza (Zabalza, 1988), así como las características de la asignatura (Burton, 2000; Pérez, 2013), éste último señala que existen competencias pedagógicas, metodológicas, académicas, y comunicativas.

$\mathrm{Al}$ ser un estudio multidimensional, se asegura que podamos entretejer los hilos de la vida del hombre que es persona humana (López, 2013), las dimensiones siguientes son el sistema de creencias y los valores.

En lo que se refiere al sistema de creencias, esta es una construcción individual-familiar-social que hace del hombre lo que es (Sartre y Valmar, 1993), este sistema de creencias es el que crea dogmas para esclavizar al sujeto, siendo en el seno de la familia donde se entretejen los primeros hilos del sujeto, muchos argumentan que los libros hacen libres a los hombres y en cierto modo tienen razón, los hace romper los eslabones que los sujetaba y los sujeta a nuevas cadenas, es un caminar sin fin. Dentro de este sistema de creencias, el sujeto que es docente transita por una verdad que es ideológica y por supuesto política y religiosa, siendo estas constitutivas en la vida escolar de estos maestros, ya que, todo acto educativo es un acto político (Meza, 2009). 
La dimensión moral y los valores axiológicos son consideradas como la tercera arista de esta búsqueda, las dos se encuentran implícitas en la educación, forman parte de su praxis, ya que la educación en sí tiene un carácter axiológico, el proceso educativo se constituye en el generador de la práctica de valores, estos valores cambian dependiendo del contexto sociocultural en que se desarrolla el proceso educativo (Figueroa, 2003). Es el docente el encargado de inculcar la práctica de valores en sus alumnos, por ello Ortega y Mínguez (2001) insisten en que los valores son afectados por la escasa moral de algunos sujetos.

Por su parte, Aguirre (2014) aborda la formación del profesorado desde lo moral (ético), genera una categoría de análisis, muchas veces obviada por el Alzheimer histórico de la sociedad, ya que en realidad si "existen aspectos morales que inciden favorablemente en el aprendizaje" (p. 2), es porque hay una buena enseñanza y por tanto profesores que se quedan en el constructo subconsciente del sujeto cognoscente para toda su vida. Flores (2018) estudia la dimensión ética del profesorado tratando de comprender aquellas dimensiones éticas que configuran las prácticas de enseñanza de profesoras universitarias que en su momento fueron designadas como memorables por sus estudiantes, en el estudio, Flores (2018) hace una caracterización In vivo con estas docentes y en esa fase nos podemos percatar de esos sistemas de creencias y valores como abonos intrínsecos en la vida docente de las maestras.

En Asia y Europa, (Arikan, Taser, y Sarac-Suzer, 2008; Werbinska, 2009; Huang, 2010; (Kourieos y Evripidou, 2013) investigaron en relación con el éxito del profesor de inglés, sus características, y roles dentro de la enseñanza de la asignatura como lengua extranjera. Arikan, Taser, y Sarac-Suzer (2008) descubrieron los atributos de un docente efectivo: amigable, entusiasta, creativo y de buen genio sin importar su género; en otras palabras, un docente tolerante en esta generación de los milennials (Walzer, 1998; Cabedo, 2006; Rodríguez, Martínez, y Rodríguez, 2017).

Huang (2010) descubrió algunas características especiales: conocedora de su materia, responsable, entusiasta, paciente y amable. Mostró que utilizaba métodos de enseñanza de acuerdo con las necesidades de sus alumnos y los entrenaba a aprender en una forma comunicativa y de modo independiente. Ella se mostraba como una amiga, dándoles apoyo a sus estudiantes en todo momento, poniendo en práctica la ética de la compasión y la teoría del capital de las caricias (Poch y Vicente, 2010; Rovira, 2015) y también como una líder entre sus colegas (Juul, 2017).

Álvarez, Porta y Sarasa (2011) realizaron un estudio de las buenas prácticas y formación del profesorado de inglés, a través de sus relatos de vida se evidencia la influencia de sus mentores en las diferentes fases del crecimiento personal e intelectual de estos profesores (incluido un profesor de inglés el cual permite dejar huellas a sus alumnos y además conformar una identidad profesional). Los maestros huellas o memorables son aquellos que generan buenas prácticas de enseñanza. Los autores concluyen que las buenas prácticas están relacionadas con el profesor en términos de: su claridad expositiva, preocupación, responsabilidad, esfuerzo y compromiso y desafíos con su enseñanza, creación de ambientes favorables, confianza en alumnos, constantes oportunidades de aprendizaje, flexibilidad con firmeza, justicia y finalmente describen como estos buenos docentes construyen conciencia sobre el lenguaje en una comunidad discursiva dentro y fuera del aula. 
En Ecuador, Chán et al. (2018) caracterizaron el perfil docente en una universidad privada, identificaron las competencias y desarrollaron diferentes propuestas de un modelo formativo. También, se realizaron estudios en relación con los perfiles de los docentes universitarios desde tres dimensiones: formación docente, sistema de creencias y valores (Mendieta, Pérez, y Villavicencio, 2020; Mendieta y Rivera, 2020; Mendieta, Avila y Cedeño, 2021).

En cuanto a los profesores universitarios de inglés y los perfiles que deben tener, existe un vacío el cual hay que llenar (Universidad Católica de Santiago de Guayaquil, 2018; (Universidad Nacional de Educación, 2021; Universidad Técnica Particular de Loja, 2020), aunque existe un estudio que da cuenta de los perfiles de los profesores unversitarios de las carreras de educación (Alfaro Rojas y Alvarado Cordero, 2018).

\section{MÉTODO}

La investigación, según el nivel de profundización, fue de tipo exploratoria (Sampieri, Collado, Lucio, y Pérez, 2010), a partir del tipo de datos que se utilizaron fue cualitativa (Hernández Sampieri, Fernández Collado, y Baptista Lucio, 2014) y también interpretativa (Behar Rivero, 2008), tuvo un enfoque biográfico narrativo (Bolívar, y Fernández, 2001; Bolívar y Domingo, 2006). Se utilizaron las técnicas de la observación (áulica), encuestas, entrevistas en profundidad y semi estructuradas.

Se empleó las técnicas de la encuesta para la selección de los participantes (Abascal y Grande Esteban, 2005; Alvira, 2011; Grasso, 2006; Jansen, 2013). Se emplearon las entrevistas para la recolección de información (Kvale, 2019; Ruiz
Blázquez, 2015), además, la técnica de la observación (Angrosino, 2007); (Peña Acuña, 2011).

Se construyó una encuesta inicial para determinar a los docentes participantes del estudio, guiones semi estructurados para las entrevistas en profundidad y una ficha de observación para la observación áulica. Tanto la encuesta como el guion de entrevista fueron adaptados de Álvarez, Porta, y Sarasa (2011). Se realizaron observaciones áulicas de clases grabadas que los docentes compartieron, éstas fueron a través de fichas de observación para su posterior análisis e interpretación.

Los instrumentos fueron validados en tres momentos: confirmación de los guiones previamente enviados vía correo electrónico a los participantes; el segundo momento es la entrevista en sí; y, por último, se enviaron las transcripciones de las entrevistas realizadas a los consultados para que lean y editen alguna palabra que resultase un lapsus linguae o alguna muletilla que ellos consideraban que no debía ir. (Denzin y Lincoln, 2015).

Se utilizaron criterios de inclusión: Ejercer la docencia en una de las universidades participantes, querer y tener tiempo para participar del estudio. Se envió una carta de consentimiento informado por escrito a los docentes participantes.

\section{DESARROLLO Y DISCUSIÓN}

Para seleccionar la población se realizó una encuesta a los estudiantes del último año o semestre de las universidades participantes. Los estudiantes que realizaron las encuestas fueron 853, en promedio el $38 \%$ de alumnos escogió al docente huella 1 y en promedio el 14\% de alumnos escogió al docente huella 2, sus nombres se anonimizaron (codificaron con números romanos) manteniendo el principio número 24 de derecho a la privacidad 
(Asociación Médica Mundial, 2017). Los resultados fueron desagregados en las tres dimensiones de estudio, así:

La formación docente fue obtenida de sus currículos profesionales depositados en el departamento de talento humano de cada universidad con fecha de última revisión en noviembre del año 2020, estos son los resultados.

Cuando Mizell (2010) argumenta que la formación docente debe ser el acopio de experiencias formativas y continúas en un maestro, esto interpela y a la vez convoca a mirar a aquellos profesores en el Ecuador que trabajan la asignatura del idioma inglés. En el presente estudio ilusiona saber que existe un $52 \%$ de profesores que tiene formación universitaria en pedagogía del idioma, se dice significativo porque hasta hace algunos años los profesores de inglés eran personas que hablaban ese idioma, pero no tenían formación en docencia. Mediante esta información se considera que en el proceso de interaprendizaje los estudiantes reciben una transposición didáctica pertinente (Gómez, 2005; Chevallard, 2015), ya que en las universidades se recibe formación en la adquisición de habilidades pedagógicas, didácticas y destrezas que luego serán competencias docentes (Zabalza, 1988; Cano, 2005; Tobón, 2008; Perrenoud, 2014).

En lo que tiene que ver con la formación continua es satisfactorio conocer que existe gran responsabilidad en los maestros por renovarse permanentemente, en la mayoría de los casos sobrepasan las mil horas entre cursos y seminarios de más de veinte horas académicas; en posgrado han realizado sus estudios en docencia universitaria y del idioma inglés, todos salvo uno de ellos que su estudio fue en otra área profesional. Podemos notar claramente que nuestros maestros realizan una de las funciones sustantivas de la academia, esta es la investigación, así tenemos que tener un promedio de 3 publicaciones resultados de sus investigaciones en los últimos cinco años.

En relación con sus prácticas docentes, fue de gran apoyo los vídeos que facilitaron y esta modalidad virtual acercó a sus clases, se utilizaron una ficha de observación que fortalece el criterio que se obtuvieron de los docentes de inglés en la universidad ecuatoriana, en realidad cumplen satisfactoriamente aquellas competencias que de orden general mencionan (Zabalza, 1988; Cano, 2005; Tobón, 2008; Perrenoud, 2014). Se coincidió que los profesores poseen esas características por las que sus estudiantes los nombraron maestros huellas. Los maestros poseen destrezas y habilidades blandas, coincidiendo con (Wats y Wats, 2019) en que son características de comunicación asertiva, pensamiento crítico, ética y moral las que les han permitido tener éxito.

Para conocer sobre los maestros, se realizaron guiones de entrevistas en profundidad semi estructuradas, el instrumento fue diseñado abordando multidimensionalmente al sujeto, las preguntas acercan a sus hilos familiares, sociales y escolares; su sistema de creencias en cuanto a sus inclinaciones políticas e ideologías religiosas y espirituales; y valores.

Los maestros de inglés respondieron a todas las preguntas. En cuanto a su hilo histórico, se tiene que realizar por separado los relatos de estos profesores; sin embargo, se quiere dejar algunas historias que interpelaron profundamente la sensibilidad de los autores de la presente investigación, tal es el caso del profesor $\mathrm{V}$, de origen humilde, quien para poder estudiar la licenciatura de inglés tuvo que emigrar a una ciudad a nueve horas de su pueblo 
natal. Entre sus relatos conmovió el hecho de que en algunos días no tenía para alimentarse e iba a un restaurante que quedaba en una esquina y le pedía a la dueña que le permita lavar los platos por una merienda, o la vez en que los amigos con los que compartía habitación (cinco personas en un cuarto de habitación) se fueron de vacaciones y dejaron con llave ese cuarto y tuvo que pernoctar en la calle tres noches. A pesar de esto, él nunca perdió la fe en que iba a triunfar cosa que así ocurrió.

Resultó un retroceder en el tiempo cuando relataban que, en sus escuelas, hubo un profesor al cual, a pesar de los años, aún los recuerdan con profundo afecto y cariño. En este momento de la entrevista, se devela que los autores del estudio también recordaban a un profesor que les marcó de por vida con sus buenas prácticas pedagógicas y humanas. En más de una ocasión se interrumpió a los entrevistados para "también" relatar nuestras experiencias con esos "nuestros profes". Esta parte de la entrevista ha sido mirar por el retrovisor de las vidas, de los autores del presente estudio, esa historia que estaba presente y surgió para sentir desde la otredad de la entrevista que se mutaba, es decir, los relatos de vida atravesaron a los que escribieron este artículo.

El profesor III comentó que su maestra de segundo grado de nombre María, todas las mañanas lo esperaba en una esquina para llevarlo a la escuela y le regalaba una galleta, "ese era nuestro ritual de buenos días, ella me daba una galleta y yo le daba un abrazo". En este relato sale a luz que, a pesar del tiempo esa ética de la compasión que su maestra le brindó permanecía intacta en el recuerdo del entrevistado y que es por eso por lo que él decidió ser maestro. Comentaba que él regala galletas a sus estudiantes en la universidad, a veces sus compañeras le bromean diciendo "llegó el galletero" pero sabe que lo dicen con cariño porque conocen su historia. Para terminar, relata que en una ocasión estaba en la playa con sus hijos y que de pronto se acercó una señora y le dijo "usted es el profesor que le regala galletas a mi hija en la universidad, gracias por hacer que mi hija aprenda a ser solidaria con las personas, ella en navidad regala una cesta de comida a algún niño que elige de los muchos que están trabajando en las calles” De pronto apareció Astrid, su ex estudiante, lo abrazó y le dijo "Míster qué gusto de volver a verlo, le conté a mi mamá que usted estaba aquí y se me adelantó".

El sistema de creencias está fuertemente influenciado por los requisitos experienciales de la infancia, los maestros están constituidos por esos requisitos (Sartre y Valmar, 1993) aunque tienen identidad propia (Carpio, 2004). Ellos prefieren no hablar de política, dicen que la política le hace mucho daño a la educación; sin embargo, se desvisten en sus ideologías en cuanto su voz se deja escuchar, es esta apertura, este visitar los territorios políticos de los profesores, los que nos permiten otorgarle a esa voz el derecho a sentir y decir lo que no quieren decir (pero lo piensan) y que es en sí lo que los representa. Son relatos, vivencias, enunciaciones reflexivas debidamente contextualizadas en el tiempo y espacio que se convierten en poderosos dispositivos de crítica y proposición de cambio del viejo statu quo político que habitan con desgano en nuestros "teachers". Lo que más se repite en sus relatos son las discrepancias con la burocracia como ejercicio docente que les ha tocado incursionar, ahora, a más de ser docentes, son secretarios.

Los profesores están seguros de que es muy limitado el espacio que les han otorgado para transitar, como señala el maestro $\mathrm{X}$ en su 
universidad a los profesores de inglés les tienen apartados de las reuniones generales. Los planes de estudio los tienen que realizar bajo otras consignas y sus clases están recargadas de situaciones que se alejan de la pedagogía y su didáctica, señala que ahora hay que presentar tantas evidencias que se trabaja hasta 16 horas diarias; el profesor VII relató que las coevaluaciones, son dadas por docentes que no tienen las competencias propias de este idioma, en la mayoría de los casos, no saben ni hablar el inglés y nos preguntan "Miss en el cuadro de trabajo autónomo, ¿qué dice que va a hacer?".

Por último, coincidieron en indicar que las políticas internas de sus universidades no convocan a la formación de estos, es más, son firmes al señalar que existe un divorcio entre las leyes de la educación superior. El docente XII señala que en el artículo seis literal "c" de la Ley Orgánica de Educación Superior (LOEI, 2010) dice que tienen derecho a acceder a cargos directivos, pues eso no se da. Por último, en el literal " $h$ " se lee claramente que tiene derecho a recibir capacitación acorde a su formación profesional, a pesar de ello, cuando desean estudiar doctorados en su área, es casi imposible que las instituciones les cumplan con ese derecho. Como se puede ver, esta dimensión es influyente en la vida de los docentes, Ortiz (2015) señala que un profesor que manifieste que no tiene ideología o, que su ideología no está presente en el aula de clase, se engaña o trata de engañar, de igual manera, Mera (2006) sostiene que todo sujeto está políticamente influenciado y de igual forma influencia a sus estudiantes.

En cuanto al plano religioso, los maestros aseguran creer en Dios, aunque pertenecen a distintas religiones, se dicen no ser practicantes salvo el profesor V que es de la Iglesia de Jesucristo de los Santos de los últimos Días. Algo que llamó la atención es la espiritualidad con que evocan a la paz interior, la felicidad y el amor. Coinciden que la mejor forma de estar en paz con Dios es haciendo el bien, siendo buenas personas y con ello se vislumbra otra vez el plano espiritual.

En el tema de la espiritualidad, la docente II reflexiona y nos cuenta que estaba perdida, creía que el mundo no era un lugar para ella, relató: "una amiga me dijo, vete a la iglesia de la Concepción, allí en una esquina está el santísimo, ese lugar es mágico, te aseguro que, si vas, tu vida encontrará el sentido que anda buscando", así lo hizo. Comentó que fue a ese lugar y que se sentó frente al santísimo, no sabe el tiempo que permaneció en ese lugar, tal vez fueron cuatro o cinco horas, puso énfasis en decir que "cuando salí era de noche, en casa estaban preocupados por mi ausencia", hace un alto a su relato, piensa, como buscando algo pierde su mirada en el firmamento, regresa y suspira al tiempo que esboza una sonrisa que no le alcanza a llegar a los ojos y con un tono de voz apacible dice "a partir de esa noche, siento una paz en mi interior que no es posible de explicarles por qué no me entenderían, comprendí que estamos en este mundo con la única tarea de ser felices y estar en paz con nosotros". Se vuelve a detener y con firmeza y ternura concluyó "Hubo una voz silenciosa que me decía dulcemente ¡Te amamos!”

En relación con los valores, Ortega y Mínguez (2001) insisten en que, es la condición que posee el ser humano y que interviene en la cotidianidad, argumenta además que, los valores son afectados por la escasa moral de algunos sujetos. Esta moral es una construcción individual y colectiva, es aquella que atañe a las elecciones personales de un individuo, aceptando que forma parte de una tendencia moral colectiva que lo presiona y controla, pero a la que también puede oponerse en su fuero interno.

El maestro XI comentó que la moral es histórica contextual, temporal, dialéctica, señala 
que a sus estudiantes les dice que "aquello de que lo que para nosotros es inmoralmente aceptable, en otras culturas es moral o viceversa" señala que a sus alumnos les puso el ejemplo de que, ¿será moral que un hombre tenga 7 mujeres? Porque en Marruecos sí es normal y moralmente aceptable. Para este maestro la moral pertenece al sujeto y es del sujeto, es individual en el hombre, pero este se ancla a una construcción social, es decir a la normativa de la sociedad.

El profesor IX pone el dedo en la llaga al decir, como principio de vida está el "no matarás" ¿verdad? Desde hace un tiempo atrás se practica el aborto, es una decisión individual de la mujer, por alguna circunstancia lo realiza y ella sabrá por qué lo hace. Yo no cuestiono el acto, no tengo derecho para hacerlo. Cuando un gobierno aprueba el aborto, como un acto de legalidad, eso va en contra de mi moralidad individual, aunque la normativa moral social lo apruebe, no puedo aceptarlo y yo no soy un santo, en ello está reflejada la moral individual y la moral social. No porque la normativa moral diga que eso es correcto, mi moral individual lo va a aceptar como correcto, aunque mire los toros de lejos y aunque ya no cuestione porque la normativa moral y el gobierno así lo dispuso, no hablo de este país y ventajosamente no se da esto, repito, no cuestiono el acto individual del aborto, que por algún motivo lo hacen, hablo del acto colectivo de aprobación del aborto.

El docente VII considera que la ética y la moral pertenecen a un conjunto y no están relacionadoscon el profesionalismo. Él siempre trata de comportarse de manera ética siendo muy profesional con sus alumnos, incluso cuando bromea con ellos ya que es un docente lleno de humor en sus clases, sabe separar las cosas muy bien. Él no solo enseña inglés a sus alumnos. Él explicó que los forma y que aprendió eso a lo largo de su vida: una institución educativa forma personas éticas y siendo parte de ella debe de reflejarse en ella sobre todo dando ejemplo vívido. Un día, por ejemplo, una alumna quería que la pasen de año y le dijo: "Señor, si usted me ayuda, nadie lo sabrá" y el docente le contestó "sí, pero yo lo sabré y me recordarás por eso, y pensarán que eso está bien, porque es un pequeño favor". Considera que los alumnos deberían estudiar filosofía y ética. Contó que trabaja en una universidad muy técnica $\mathrm{y}$ sus alumnos tienen la idea de que ser buenos en el aspecto técnico y científica, significa ser un buen profesional, pero no es así. Adicionalmente, siempre incluye preguntas de análisis sobre asuntos moralmente cuestionables. Si bien usan gramática aprendida su intención es que sepan expresar su opinión, ser respetuosos con los buenos de vistas ajenos y tal vez porque no aprender algo nuevo.

El docente II relató que existen valores que forman parte de su vida como profesor, señala que la ética es su principal siembra, que como persona posee un conjunto de valores que hacen de él lo que es y a la vez los ha cultivado con el paso de los años, estos valores son la honestidad, el respeto, la disciplina y la justicia, manifiesto que, aunque hay otros valores como el amor y la compasión, para él sin ética no hay buena docencia.

En el marco de lo anterior, se coincide con Aguirre (2014) cuando menciona que existen aspectos morales que inciden favorablemente en el aprendizaje, por supuesto que sí, eso nos lo han demostrado los profesores con sus anécdotas con sus profesores en sus años escolares. Son estas minucias de la vida diaria escolar las que permite asegurar que existe una dimensión ética de la enseñanza, tal como lo menciona Flores (2018). Por último, existe un hilo vital que una de las buenas prácticas de los docentes de inglés en las universidades con los que una vez fueron sus maestros. Se concuerda que los maestros huellas de inglés despliegan dispositivos 
de enseñanza untados con la ética de la compasión (Poch y Vicente, 2010) y la teoría del capital de las caricias (Rovira, 2015).

La construcción del perfil del profesorado de inglés en las universidades del Ecuador es algo complejo pero fascinante de hurgar. Cada docente tiene relatos de vida únicos e irrepetibles, una sólida formación profesional, creencias muy inherentes a cada uno, valores y características propias que los hacen ser esos docentes memorables y que dejan huellas en sus alumnos.

Es preciso destacar que para adquirir una lengua extranjera y ser docente de inglés en Ecuador se requiere de un sacrificio adicional y de haber invertido muchos años de estudio y mejora continúa con poco apoyo de sus instituciones al momento de querer capacitarse dentro y fuera del país. El docente de esta lengua posee las mismas competencias que otros docentes universitarios. Sin embargo, hay un sello particular y es que en su afán de crear un ambiente agradable y de confianza para con sus alumnos intenta planear sus clases, cada actividad minuciosamente de un modo lúdico, sencillo, amigable incluyendo un elemento de motivación intrínseca que despierte en el alumno su curiosidad y sus ganas por aprender. Los alumnos y colegas los reconocen como diferentes, más alegres, dinámicos, interactivos y que esperan la clase de inglés "para relajarse un poco". Estos mismos alegan que los "teachers" son más pacientes, empáticos y compasivos que los maestros de otras áreas. La mayoría de estos docentes no son nativos y esto les permite de uno y otro modo ponerse en el zapato de sus alumnos y lograr ser mejores guías en el proceso de aprendizaje. También fue destacado que con el mismo amor y compasión que tratan a sus alumnos igualmente les exigen intentando ser justos en el proceso de evaluación. Un docente dijo que era otra persona e inclusive otro expresó que era como un actor en el salón y es que este docente intenta hacerse uno con sus alumnos para lograr llegar a ellos.

\section{CONCLUSIONES}

Se logró interpretar el perfil del profesorado universitario de inglés en Ecuador con una mirada socio histórica a partir de las narrativas y experiencias de los propios docentes en relación con las aristas que se develan a continuación.

Los docentes tienen una sólida formación profesional y su estatus como docentes es el resultado de sus continuas asistencias a seminarios, talleres, maestrías, doctorados, entre otros. Esto se refleja con las investigaciones que han realizado y las publicaciones que ellos tienen en revistas y libros a nivel internacional, el cumplir con esa función sustantiva de la academia ecuatoriana les hace ser acreedores del respeto de quienes los conocen y nos adherimos a ese respeto y admiración.

La mayoría de los docentes han desarrollado competencias educativas sobre planeación, elección de estrategias y técnicas de enseñanza, interacción constante con los alumnos y entre ellos y diferentes tipos de evaluación. La enseñanza del inglés ofrece a los profesores metodologías y técnicas que debido a su carácter internacional van un paso delante de las otras asignaturas de educación superior en este país.

Algunos alumnos llegan a las universidades sin buenas bases en este idioma. Esto hace que resalte la tarea de estos docentes que logran motivar a sus estudiantes desde su pasión por enseñar vivenciada en sus dinámicas de la clase, el trato amigable, paciente, empático y a la vez estricto buscando ser siempre éticos, justos y profesionales, esto se pudo comprobar en la observación de sus clases. Los alumnos de los maestros huellas expresan como de uno u otro modo estos docentes lograron llegar 
a sus adentros para dejar esta semilla de querer aprender el inglés.

Los docentes presentan una impronta ideológica muy arraigada y poco visibilizada en su ejercicio docente, aunque todos plantearon no hablar de política, al final se notó que estaba arrimados a una vera ideológica en cuanto a la política, principalmente en el campo de las políticas educativas. Por otro lado, la religión es un abono que les llena y les hace ser y ejercer la docencia basada en aquellos principios de sus respectivas religiones, pero, lo que más denotan los profesores es su espiritualidad, la cual estuvo presente en cada uno de sus relatos, en definitiva, el sistema de creencias de los maestros es constitutivo y está presente en cada uno de ellos.

Los valores como el respeto, tolerancia, amor, otredad, honestidad, la moral y la ética están formando parte de la vida de los docentes de inglés, en todas las entrevistas sentimos que estos principios de vida se entraman unos con otros para hacer un bordado color esperanza que nos hace tener fe en que los estudiantes reciben clases con estos maestros están bajo el manto de los valores y las buenas enseñanzas del idioma. El perfil docente es una construcción multidimensional, personal e inédita, pero a la vez, posee características heterogéneas.

Los maestros huellas de inglés en Ecuador mostraron sencillez en todo el proceso de la investigación, por eso nos quedamos con el sabor a ajenjo, ese ajenjo que repara el alma al haber sido parte de este proyecto, de haber escuchado de los "Teachers" las vivencias que nos convidaron con generosidad, así también, nos queda el amargo de la despedida, nos hubiese gustado que no se termine aún, seguir escuchando sus relatos de vida para interpretar y comprender que esa forma tan peculiar de hacer docencia.

\section{REFERENCIAS}

Aguirre, J. (2014). La ética y valores morales en la enseñanza universitaria desde la perspectiva de los estudiantes: conjunción de intelecto, compromiso, afecto y pasión en los profesores memorables. Entramados: educación y sociedad, 12. http://fh.mdp.edu.ar/revistas/index.php/ entramados

Abascal, E., y Grande Esteban , I. (2005). Análisis de encuestas. Madrid: Esic editorial. doi:84-7356420-0

Alfaro Rojas, G., y Alvarado Cordero, S. (2018). El perfil de profesores universitarios de universidades públicas y privadas en la carrera de Educación. Revista Actualidades investigativas en educación, 18(2), 1-21. doi:https://doi. org/10.15517/aie.v18i2.33161

Álvarez, Z., Porta, L., y Sarasa, M. C. (2011). Buenas prácticas docentes en la formación del profesorado: Relatos y modelos entramados. Profesorado. Revista de Curriculum y Formación del Profesorado, 229-240. doi:1989-639X

Alvira. (2011). La encuesta una perspectiva general metodológica. Madrid- España: Centro de investigaciones Sociológicas. Primera edición https://ddd.uab.cat/pub/caplli/2016/163567/ metinvsoccua_a2016_cap2-3.pdf

Angrosino, M. (2007). Etnografía y observación participante en investigación cualitativa. Madrid: Morata. doi:ISBN: 978-84-7112-695-5

Arikan, A., Taser, D., y Sarac-Suzer, H. (2008). The Effective English Language Teacher from the Perspectives of Turkish. Online Submission, Education and Science, 10. https://files.eric. ed.gov/fulltext/ED506217.pdf

Asociación Médica Mundial. (2017). Declaración De Helsinki De La Amm - Principios Éticos Para Las Investigaciones Médicas En Seres Humanos. Obtenido De Declaración De Helsinki De La Amm - Principios Éticos Para Las Investigaciones Médicas En Seres Humanos: https://acortar.link/Omxyu

BBC Worldwide World. (2020). Los países que hablan inglés y su porcentaje en el mundo. https://acortar.link/tI89O 
Behar Rivero, D. (2008). Metodología de la Investigación. Lima: Shalom. doi:ISBN 978959-212-783-7

Bolívar, A., y Domingo, J. (2006). La investigación biográfica y narrativa en Iberoamérica:Campos de desarrollo y estado actual. Forum:Qualitativesocialresearch, 7(4), 1-43. doi:1438-5627

Bolívar , A., y Fernández, M. (2001). Investigación Biográfico-narrativa En Educación. Madrid: La Muralla.

Burton, J. (2000). Leaving from teaching practice: a case study approach. Learning from teaching, 18. file://C:/Users/PC/Downloads/Publisher\%20 version\%20(open\%20access).pdf

Cano, E. (2005). Cómo Mejorar Las Competencias De Los Docentes (Primera ed.). Barcelona: Editorial GRAÓ, de IRIF, S.L. doi:ISBN 10: 847827-398-0

Carpio, A. (2004). Principios de la filosofía (segunda ed.). Buenos Aires, Argentina: Glauco. doi:ISBN: 950-9115-01-0

Chán, M. M., Goded, P. A., y Sacaluga, C. (2018). Validación de cuestionario para Caracterización del Perfil Docente (CAPED) de la Universidad Tecnológica ECOTEC. Revista Espacio, 39(15).

Chevallard, Y. (2015). La transposición didáctica. Del Saber Sabio Al Saber Enseñado. AIQUE.

Consejo de Educación Superior. (2017). Reglamento De Regimen Academico Consejo. https://www. ces.gob.ec/lotaip/2018/Enero/Anexos\%20 Procu/An-lit-a2-Reglamento\%20de\% 20 R\%C3\%A9gimen\%20Acad\%C3\%A9mico.pdf

Consejo de Educación Superior. (2019). Reglamento De Regimen Academico Consejo. Https:// Acortar.link/UtKjY

Denzin, N., y Lincoln, Y. (2015). Métodos de recolección y análisis de datos: Manual de investigación cualitativa (Vol. IV). Barcelona: GEDISA. doi:978-84-9784-311-9

Flores, G. (2014). Bios, poder y política. Una perspectiva desde el enfoque decolonial crítico de la Educación. Revista de Educación, 7, 427444. doi:1853-1326
Flores, G. (2018). Dimensión ética de la enseñanza. Un estudio interpretativo de las prácticas de profesores memorables de la Facultad de Humanidades de la Universidad Nacional de Mar del Plata. Revista de Educación, 14, 175178. https://acortar.link/p8af1

Gómez, A. (2005). La Transposición Didáctica: Historia De Un Concept. Revista Latinoamericana de Estudios Educativos, 83115. Https://goo.gl/937ZCo

Grasso, L. (2006). Encuestas. Elementos para su diseño y análisis (Primera edición ed.). Argentina: Encuentro Grupo editor. doi:98723022-3-5

Hernández Sampieri, R., Fernández Collado, C., y Baptista Lucio, M. d. (2014). Metodología de la investigación (sexta ed.). México: McGRAWHILL. doi:ISBN: 978-1-4562-2396-0

Hervis, E. (2018). El desempeño del docente como factor asociado a la calidad educativa en América Latina. Revista Educación, 42(2), 717739. doi:ISSN: 0379-7082

Huang, Z. (2010). What Makes a Successful EFL Teacher in China? A Case Study of an English Language Teacher at Nanjing University of Chinese Medicine. English language teaching, 8. https://files.eric.ed.gov/fulltext/EJ1081836.pdf

Jansen, H. (2013). La lógica de la investigación por encuesta cualitativa y su posición en el campo de los métodos de investigación social. Paradigmas: Una Revista Disciplinar de Investigación, 5(1), 39-72. doi:1909-4302

Juul, J. (2017). Líderes de la Manada. (M. L. Vera Soriano, Trad.) Barcelona: HERDER. doi:ISBN: 978-84-254-3850-9

Kourieos, S., y Evripidou, D. (2013). Students' Perceptions of Effective EFL Teachers in University Settings in Cyprus. English Language Teaching, 16. https://files.eric.ed.gov/fulltext/ EJ1078477.pdf

Kvale, S. (2019). Las entrevistas en investigación cualitativa. s/n: MOrata. doi: 978-84-7112-630-6 
López, J. (2013). Fernando Rielo. Concepción mística de la antropología. (J. M. López, Ed.) Madrid, Madrid, España: Fundación Fernando Rielo. doi:ISBN <. 84-86942-87-X

Mendieta Toledo, L., Avila Redrobán, V., y Cedeño Rivera, K. (2021). Laura Mariscal Touzard y su historia de vida. Guayaquil: Crisálidas. doi:9789942-40-454-1

Mendieta, L., Pérez, L., y Villavicencio, V. (2020). Pedro Rizzo y su historia de vida. Guayaquil: CIDE. doi:978-9942-802-78-1

Mendieta, L., y Rivera, E. (2020). Katuska Cepeda y su historia de vida. Guayaquil: CIDE. doi:9789942-802-73-6

Mensias Pérez, N. M. (2016). Perfil de egreso en la formación de profesores de inglés en el desempeño de práctica preprofesional docente 4 de la Carrera de Inglés, Facultad de Filosofía, Letras y Ciencias de la Educación, de la Universidad Central del Ecuador periodo lectivo 2015 - 20169. Obtenido de: https://scholar.google.es/scholar?hl=es\&as_ $\mathrm{sd} \mathrm{t}=0 \% 2 \mathrm{C} 5 \& \mathrm{q}=\mathrm{el}+\mathrm{profes}$ or a d o + universitario+de+ingles+ ecuador.+perfiles\&btnG=

Mera, C. (2006). La dimensión política y la dimensión académica de la universidad. Revista Habitat Inclusivo. Https://goo.gl/cjpyWt

Meza, L. (2009). Elementos de pensamiento crítico en Paulo Freire: Implicaciones para la educación superior. Revista digital: Matemática, Educación, Internet, 10(1), 1-11.https://doi.org/10.18845/ rdmei.v10i1.1975

Mizell, H. (2010). Why Professional Development Matters. Learning Forward. 504 South Locust Street, Oxford, OH 45056

Murillo, G. (2020). Vidas narradas en educación (Seminario Miradas Transatlánticas). s/c, s/p, s/P. https://acortar.link/aghjz

Ortega, P., y Mínguez, R. (2001). Los valores en la educación. Barcelona: Ariel Educación. doi:ISBN: 84-344-2640-4

Ortiz, A. (2015). Docencia universitaria. Barranquilla: Distribooks editores. doi:doi:978-958-33-8821-7
Peña Acuña, B. (2011). Métodos Científicos de Observación en Educación. Madrid: Visión Libros

Pérez, V. (2013). El profesor de idiomas: sus cualidades y competencias. Revista communication, 12. Obtenido de https://acortar. link/NRmuv

Perrenoud, P. (2014). Diez Nuevas Competencias Para Enseñar. Barcelona: GRAÓ. doi:ISBN: 97884-9980-542-9

Poch, C., y Vicente, A. (2010). La acogida y la compasión Acompañar al otro. En J. C. Mélich Sangra, y Á. Boixander, Los márgenes de la moral (pág. 151). Barcelona: Graó. doi:ISBN: 978-84-7827-909-8

Rodríguez, K., Martínez, A., y Rodríguez F. (2017). Estudio empírico sobre los valores democráticos de tolerancia y respeto en la generación milenaria. En Justicia, 31, 135-150. doi:http:// dx.doi.org/ 10.17081/just.22.31.2603

Rovira, A. (2015). Álex Rovira habla de la Economía de caricias, el efecto Pigmalión, la gestión de los equipos. Tenerife, Gran Canarias, España. https://www.youtube.com/results? search_ query=alex+rovira

Ruiz Blázquez, J. J. (2015). Las entrevistas en profundidad y la biografía. Revista San Gregorio, 48-55. doi:1390-7247

Sampieri, R. H., Collado, C. F., Lucio, P. B., y Pérez, M. D. L. L. C . (2010). Metodología de la investigación. México: Mcgraw-hill.

Sartre, J. P., y Valmar, J. (1993). El ser y la nada. Barcelona: Altaya. https://acortar.link/7oOxW

Secretaría de Educación Superior, Ciencia, Tecnología e Innovación. (2010). Ley Orgánica De Educación Superior, LOES. Https://acortar. link $/ \mathrm{jHCoO}$

Tobón Tobón, S., Rial Sánchez, A., Carretero Díaz, M. A., \& García Fraile, J. A. (2006). Competencias, calidad y educación superior (No. 378.1 C737c). Cooperativa Editorial Magisterio 
Tobón, S. (2008). La formación basada en competencias en la educación superior: el enfoque complejo. Guadalajara: Universidad Autónoma De Guadalajara. https://goo.gl/ uVVCyD

Unesco. (1995). Documento de política para el cambio y el desarrollo en la educación superior https://unesdoc.unesco.org/ark:/48223/ pf0000098992_spa

Universidad Católica de Santiago de Guayaquil. (2018). Carrera de Pedagogía de los Idiomas UCSG. Obtenido de Carrera de Pedagogía de los Idiomas - UCSG: https://www.ucsg.edu.ec/ art/c240400/\#inegres

Universidad Nacional de Educación. (2021). Pedagogía de los Idiomas Nacionales y Extranjeros. Obtenido de Pedagogía de los Idiomas Nacionales y Extranjeros: https:// unae.edu.ec/oferta/pedagogia-de-los-idiomasnacionales-y-extranjeros/?portfolioCats $=12$

Universidad Técnica Particular de Loja. (2020). Carrera de Pedagogía de los Idiomas Nacionales y Extranjeros. Obtenido de Carrera de Pedagogía de los Idiomas Nacionales y Extranjeros: https:// www.utpl.edu.ec/carreras/idiomas
Walzer, M. (1998). Tratado sobre la tolerancia. Barcelona: Paidós Coleción Estado y Sociedad. doi:9788449306181

Wats, M., y Wats, R. (2019). Developing Soft Skills in Students. International Journal of Learning, 10. https://www.researchgate.net/ publication/331629090

Werbinska, D. (2009). A profile of an effective teacher of English: A qualitative study from Poland. Hacettepe Üniversitesi Eğitim Fakültesi Dergisi, 36(36). Hacettepe Üniversitesi Eğitim Fakültesi Dergisi (H.U. Journal of education), 9. https://dergipark.org.tr/en/download/articlefile/87512

Zabalza, M. A. (1988). Competencias docentes del profesorado universotario. NARCEA 